\title{
Strangulated sigmoid volvulus with severe hypothyroidism: A disastrous combination, our experience
}

\author{
C.R. Praveen ${ }^{1}$, B.N. Patowary ${ }^{2}$, A. Agarwal $^{1}$, S. Kumar ${ }^{1}$, A. Karmacharya ${ }^{3}$, P. Shrestha ${ }^{3}$ \\ ${ }^{1}$ Lecturer, ${ }^{2}$ Professor and Head, ${ }^{3}$ Resident, Department of Surgery, College of Medical Sciences, Bharatpur, Chitwan, Nepal
}

\begin{abstract}
Both sigmoid volvulus and hypothyroidism, can individually have serious repercussions in terms of morbidity and mortality. Even worst, is a combination of these two clinical entities. We report a case of this lethal combination, which had gone into myxedema coma after emergency surgery for strangulated sigmoid volvulus. Patient could be saved because of early identification and meticulous management. It is suggested to have high index of suspicion of hypothyroidism in patients of volvulus.
\end{abstract}

Key words: Combination, hypothyroidism, myxedema coma, sigmoid volvulus.

\section{Introduction}

In a patient of peritonitis with hypothyroidism, there are potential anaesthetic and postoperative complications. The most serious complications are sepsis and myxedema coma. Few postoperative findings are common to both: like difficult extubation, hypotension, hypoglycaemia and hypothermia. Hence in an undiagnosed case of hypothyroidism, these postoperative findings may be wrongly interpreted as due to sepsis. In our case all these features were present but the cause was hypothyroidism, not sepsis. Therefore, hypothyroidism needs to be ruled out in patients of volvulus who have symptoms suggestive of this condition.

\section{Case report}

66 year old male presented with generalised abdominal pain, distension and obstipation of 2

Correspondences: C.R. Praveen

E mail: praveencsr@hotmail.com days duration. He had similar complaints three months back, when he was diagnosed as a case of sigmoid volvulus with hypothyroidism. However he had spontaneous detorsion and patient was discharged on request on oral levothyroxin. However he was not compliant.

During the present episode, he presented with features of peritonitis and shock secondary to strangulated sigmoid volvulus. He had a pulse rate of 108 per minute and blood pressure of 70/ 50 $\mathrm{mmHg}$. After adequate resuscitation, erect $\mathrm{x}$-ray abdomen was done, which showed features of sigmoid volvulus but there were no features of perforation(Fig 1). His repeat thyroid profile showed high TSH levels (TSH=31.55 IU/L) with decreased FT4 (1.71pmol/l) and FT3 (1.35 pmol/ 1) levels (Table 1). As injection T3 (triiodothyronin) was not available with us, L-thyroxin tablets (400 micrograms) were given through nasogastric tube. He was put on broad spectrum 
antibiotics and injection hydrocortisone $200 \mathrm{mg}$ was given. On explorative laparotomy, massive sigmoid volvulus with patchy gangrenous segments was found (Fig 2\&3). Intra-operatively he had fall in blood pressure for which inotropic support, in the form of dopamine had to be started. In view of the gangrenous segment and the condition of the patient, resection of the segment and Hartmann's procedure (Resection of the volvulus segment and temporary end colostomy) was done(Fig $3 \& 4$ ). Post operatively he could not be extubated. He was shifted to intensive care unit (ICU) and was put on ventilator on SIMV mode. He developed hypothermia of 94 degree $\mathrm{F}$ subsequently, managed with blankets and warm intravenous fluids. After two hours he was found to be very restless. He was given $2 \mathrm{mg}$ midazolam injection for sedation, as he was unfit for extubation. He went into a state of prolonged unconsciousness after just one dose of midazolam. He also had hypoglycaemia (GRBS = $71 \mathrm{mg} \%$ ) which was corrected. Electrolytes were within normal limits. Electrolytes, blood sugars and renal parameters were closely monitored. The ICU staff was warned not to give additional sedatives in view of hypothyroidism. This state of coma remained for 18 hours. On the evening of first post operative period he regained consciousness. His vitals (temperature, pulse rate and blood pressure) were now stable. He was weaned out of ventilator. He was continued with 100 micrograms of oral thyroxin once daily. The colostomy started functioning on third postoperative day. His rest of the post-operative period was uneventful except superficial surgical site infection. He was discharged on fifteenth post-op day on oral thyroxin 100 micrograms. On follow up after 8 weeks his
TSH was 6.6 IU/1 with FT3 and FT4 within normal limits (Table 1). He underwent colostomy closure. Due to well controlled hypothyroidism he did not have any complications this time and was extubated immediately after surgery. He was discharged subsequently on 8th postoperative day.

\begin{tabular}{|c|c|c|c|}
\hline $\begin{array}{l}\text { Thyroid } \\
\text { profile }\end{array}$ & $\begin{array}{c}\text { TSH }(\mathrm{mIU} / \mathrm{ml}) \\
(0.27-4.2)\end{array}$ & $\begin{array}{c}\text { T3(pmol/L) } \\
(3.10-6.80)\end{array}$ & $\begin{array}{c}\text { T4(pmol/L) } \\
(12-22)\end{array}$ \\
\hline First surgery & & & \\
\hline $\begin{array}{l}\text { (Hartmann's } \\
\text { procedure) }\end{array}$ & 31.55 & 1.71 & 1.35 \\
\hline $\begin{array}{l}\text { Second surgery } \\
\text { (Colostomy } \\
\text { closure) }\end{array}$ & 6.6 & 3.6 & 13.10 \\
\hline
\end{tabular}

Fig 1: Erect X-ray of abdomen showing sigmoid volvulus.

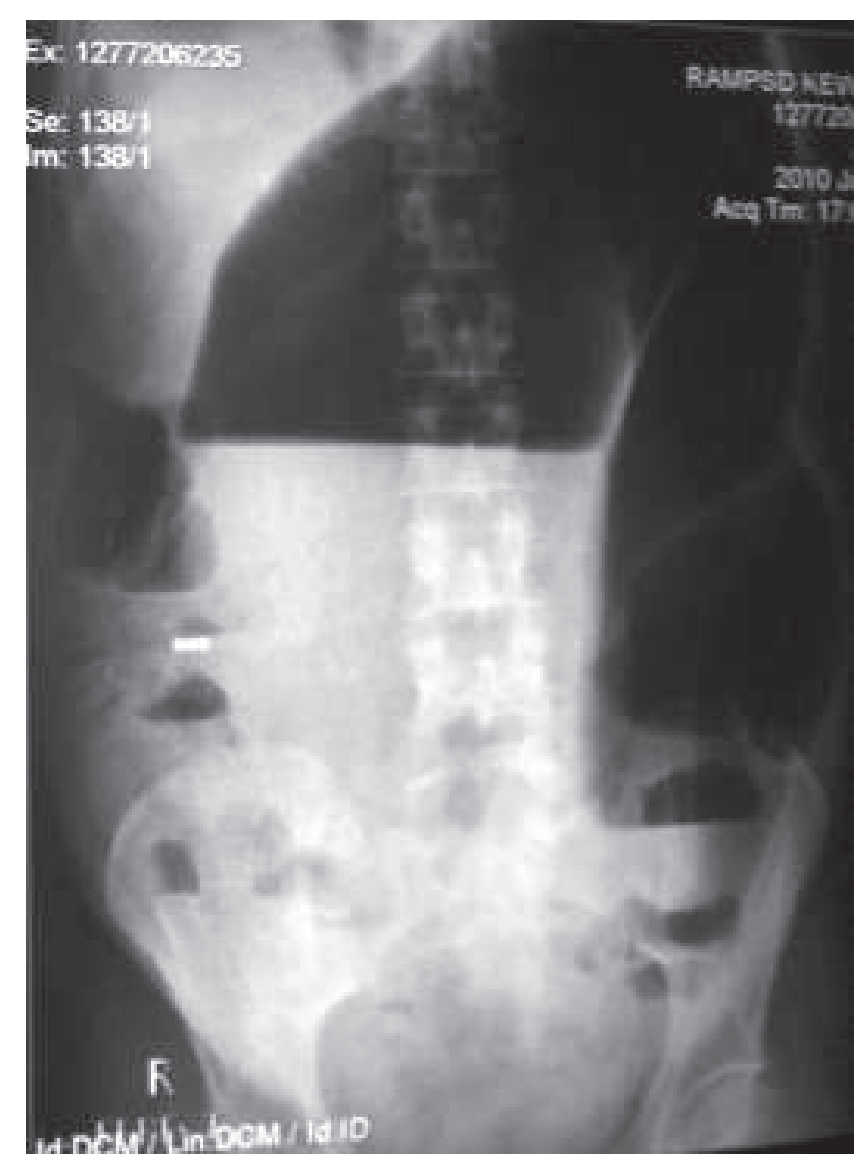


Fig 2: Intra-operative photograph showing sigmoid volvulus.

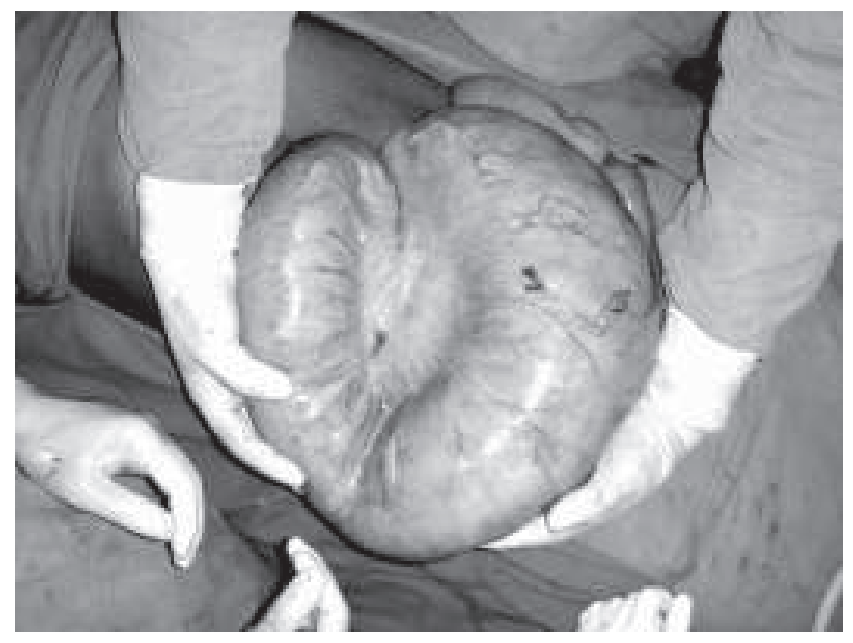

Fig 3: Resected specimen.

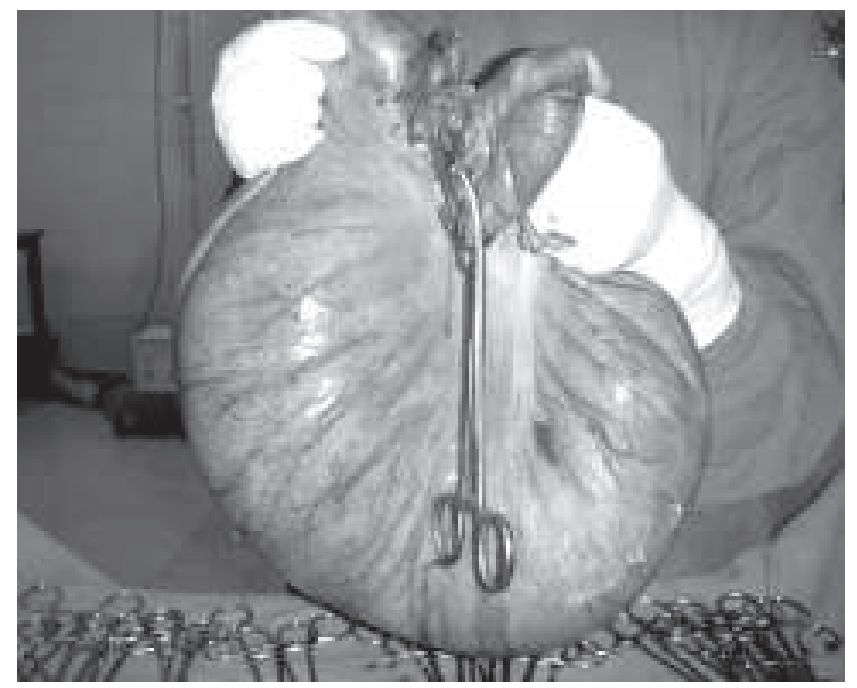

Fig 4: Temporary end colostomy.

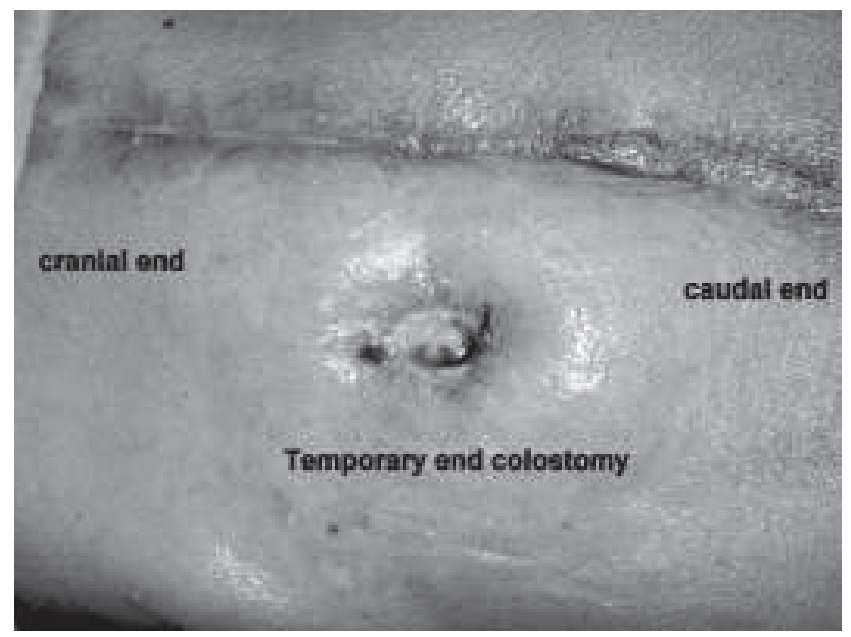

Table 1. Comparison of Thyroid profile before first surgery for volvulus (Hartmann's procedure) and second surgery for colostomy closure.

\section{Discussion}

Hypothyroidism causes constipation. Chronic constipation is a known risk factor for sigmoid volvulus. Volvulus describes the condition in which the bowel becomes twisted on its mesenteric axis, a situation that results in partial or complete obstruction of the bowel lumen and a variable degree of impairment of its blood supply. Strangulated sigmoid volvulus without surgery has mortality ranging from 18 to $75 \%$ and requires emergency surgery. ${ }^{1}$

Myxedema coma can be precipitated by surgery in patients of hypothyroidism. It can also be triggered by other stressful events like trauma, infection, myocardial infarction and following anaesthesia. Myxedema coma if not treated, has a high mortality rate (upto $80 \%$ ). However delaying emergency surgery does not improve the outcome in patients with severe hypothyroidism. ${ }^{2}$

Although thyroid hormone therapy is critical to survival, it remains uncertain whether it should be administered as thyroxine, triiodothyronine, or both. ${ }^{3,4}$ Outcome has been reported not to be influenced by the route of administration of Lthyroxine. ${ }^{5}$ However if suitable intravenous preparation of thyroxin is not available, the same initial dose of levothyroxine can be given by nasogastric tube. ${ }^{6}$ As intravenous T3 and T4 was not available with us, we gave the same loading dose of levothyroxin through the nasogastric tube. 
Sigmoid volvulus with gangrenous bowel requires resection and most would favour restoring continuity at a later stage, particularly in the elderly. Emergency resection carries a high mortality rate partly because of the poor general condition of the patient and toxaemia from necrosis. ${ }^{1}$

Treatment for myxedema coma must be initiated promptly in an intensive care unit setting. Adjunctive measures such as ventilation, warming, fluids, antibiotics, pressors, and corticosteroids, may be essential for survival ${ }^{3,4}$. Sedatives should be avoided if possible or used in reduced doses due to delayed drug metabolism. Non narcotics like ketorolac are preferred for analgesia. ${ }^{7}$ In our patient, due to his excessive restlessness a sedative was given in a small dose, which proved costly for us. Our patient had hypoventilation, hypotension, hypothermia and hypoglycaemia, which responded to thyroxin supplementation, confirming the diagnosis of myxedema coma. He also had difficult extubation and delayed drug metabolism with prolonged unconsciousness with a single dose of sedative (midazolam).

Sepsis is considered as a differential diagnosis of myxedema coma. ${ }^{2}$ It is interesting to note that difficult extubation, hypotension, hypothermia and hypoglycaemia are also reported to occur in patients with sepsis. ${ }^{8,9,10}$ Hence in a patient operated for strangulated bowel, it is natural to suspect sepsis if these findings are encountered. And this may lead the surgeon to miss the diagnosis of hypothyroidism, especially if thyroid hormone levels are not tested preoperatively or hypothyroidism is overlooked.
It is of special interest that some patients with severe biochemical hypothyroidism have only mild clinical signs, whereas others with minor biochemical changes can have quite severe clinical manifestations. ${ }^{11}$ Hence the difficulty is in diagnosing those cases who do not present with classical symptoms and signs, especially in the emergency setting. Because many patients of volvulus complain of constipation, this could be a sign of hypothyroidism. Hence we recommend routine thyroid profile analysis in patients of volvulus.

\section{Acknowledgement}

We would like to acknowledge and thank Department of Anaesthesiology and Medicine, College of Medical Sciences, for helping us in managing this patient of sigmoid volvulus with severe hypothyroidism.

\section{References}

1. T.E. Madiba, S.R. Thmoson. The management of sigmoid volvulus. J.R.Coll.Surg.Edinb. 45;1:74-80.

2. R.P. Pamela, C.P. Richard. Hypothyroidism: Myxedema Coma. John L. Atlee. Complications in anesthesia. $2^{\text {nd }}$ ed. Elsevier Health Sciences, 2007;451-3.

3. M.P. Kwaku , K.D. Burman . Myxedema coma. J Intensive Care Med. 2007; 22(4):224-31.

4. L. Wartofsky . Myxedema coma. Endocrinol Metab Clin North Am. 2006;35(4):687-98

5. H. Zulewski, B. Muller, P. Exer, et al. Estimation of tissue hypothyroidism by a new clinical score evaluation of patients with various grades of hypothyroidism and controls. J Clin Endocrinol Metab 1997; 82: 771-6.

6. J.L.L. Jameson, A.P. Weetman. Disorders of the Thyroid Gland. In: A.S. Fauci, E, Braunwald, D.L. 
Journal of College of Medical Sciences-Nepal,2011, Vol-7,No-1

Kasper, et al., editors. Harrison's principles of internal medicine. $17^{\text {th }}$ ed. New York: McGraw Hill; 2008;2229-32.

7. P.T. Prescott. Disorders of thyroid. Lubin, F. Michael. et al.. Medical Management of the Surgical Patient. $4^{\text {th }}$ Ed. Atlanta: Cambridge University Press; 2006;367-73.

8. S.I. Miller, R.J. R.J. Jr Wallace, D.M. Musher. et al. Hypoglycemia as a manifestation of sepsis. Am J Med. 1980; 68(5):649-54.
9. M.M. Hossain, S.M.A. Alim, M. Begum et al .Neuropathies in sepsis a difficult Situation to Wean from Ventilator Journal of BSA. 2008;21:53-5

10. R. Sincic, K.K. Giuliano. Reader Affirms Association Between Hypothermia and Sepsis Am J Crit Care. 2007; 16:332-3.

11. J. Beynon, S. Akhtar, T. Kearney . Predictors of outcome in myxoedema coma. Crit Care. 2008;12(1):111. 